Distaler Radius

\section{Kanülierte Gewindestifte zur Osteosynthese extraartikulärer distaler Radiusfrakturen}

Bilbrew L et al. Cannulated Self-Drilling, SelfTapping Pins for Displaced Extra-articular Distal Radius Fractures. J Hand Surg Am 2018; 43: 294.e1 - 294.e5

Instabile distale Radiusfrakturen mit dislozierten Fragmenten werden derzeit v.a. über eine Plattenosteosynthese versorgt. Die Methode führt zwar zu guten Ergebnissen, ist aber invasiver, als es möglicherweise bei manchen Patienten notwendig ist. Die Drahtosteosynthese nach Kapandji wiederum ist zwar weniger aufwendig, aber mit einer hohen Rate an sekundären Dislokationen behaftet.

Chirurgen der Universitv of Gainsville stellen nun ein fixes System zur Behandlung dieser Frakturen vor. Das T-Pin-System besteht aus einem selbstschneidenden, selbstbohrenden kanülierten Gewindestift und wurde speziell für distale Radiusfrakturen entwickelt. Bei Bedarf können damit auch einfache Frakturen des Processus styloideus radii mit Dislokation versorgt werden.

Mit dem System werden ähnlich wie mit der Plattenosteosynthese winkelstabile Verhältnisse mit guter Stabilität geschaffen, der Zugang erfolgt aber minimalinvasiv perkutan wie bei der Drahtspickung. Es sollte aber keine Abkippung der Fragmente nach palmar bestehen und mit Ausnahme einer isolierten Fraktur des Processus styloideus ulnae sollte die Ulna unverletzt sein. Bei komplizierten intraartikulären Frakturen mit Dislokation ist das System dagegen kontraindiziert. Das Gleiche gilt bei Patienten mit geringem Anspruch an die Funktionsfähigkeit der Hand, bei denen eine konservative Therapie mit alleiniger Ruhigstellung ausreicht.

Der Eingriff erfolgt meist in Regionalanästhesie und Blutleere, bei älteren, multimorbiden Patienten ist aber auch eine Lokalanästhesie mit zusätzlicher Sedierung möglich. Nach geschlossener Reposition werden die Fragmente über temporäre Drähte in situ gehalten. Dann werden über eine Hautinzision von ca. $1 \mathrm{~cm}$ zunächst ein Führungsdraht und dann der Gewindestift durch die dorsale Radiuslippe oder den Processus styloideus radii eingebracht. Die Gewindestifte sollen die gegenüberliegende Kortikalis nicht durchdringen, sondern sich an ihr abstützen. Nach Entfernung der temporären Drähte erfolgen die Hautnaht und die Anlage einer palmaren Unterarmorthese, die die volle Beweglichkeit der Finger erlaubt. Die Physiotherapie des Handgelenkes kann nach ca. 2 Wochen beginnen, nach 6 Wochen finden allmähliche Kräftigungsübungen und die Aufnahme normaler Aktivitäten statt.

Dabei ist eine gute Reposition zu Beginn für den erfolgreichen Verlauf unabdingbar. Die Länge der Gewindestifte muss mithilfe der Führungsdrähte sorgfältig bestimmt werden. Zu kurze Stifte gefährden die Stabilität, zu lange Stifte verletzen die gegenüberliegende Kortikalis. Die Stifte sollen distal auf Höhe der Kortikalis enden, was auch eine spätere Metallentfernung unnötig macht.

FAZIT

Das von ihnen verwendete T-Pin-System mit kanülierten Gewindestiften führt bei extraartikulären distalen Radiusfrakturen zu guten Ergebnissen, fassen die Autoren zusammen. Dabei ist es einfach einsetzbar und wenig invasiv, was v. a. bei den oft älteren Patienten mit mehrfachen Begleiterkrankungen vorteilhaft ist. Wenn auf eine korrekte Länge des eingebrachten Stifts geachtet wird, ist eine Metallentfernung normalerweise nicht notwendig.

Dr. Elke Ruchalla, Bad Dürrheim 